\title{
Total anomalous pulmonary venous return with intact atrial septum and associated mitral stenosis
}

\author{
WALTER G. WOLFE and PAULA. EBERT \\ Department of Surgery, Duke University Medical Center, Durham, North Carolina 27706
}

\begin{abstract}
Total anomalous pulmonary venous return with an intact atrial septum is an uncommon lesion. With associated mitral stenosis each lung has a different pulmonary flow, individual vascular resistance, and function. This represents the first reported case of this anomaly treated by total correction and mitral valve replacement. Pulmonary flow reverted to normal distribution and heart size decreased, with a reversion of vascular changes on the chest radiograph one year after operation.
\end{abstract}

Total anomalous pulmonary venous return with an intact atrial septum, an uncommon lesion, is even more unusual with associated mitral stenosis. Gilman and co-workers (Gilman, Skowron, Musser, and Bailey, 1957) reviewed their experience with total anomalous venous return and presented one case with intact atrial septum and associated mitral stenosis. Both lesions were corrected at the same operation. That same year, Ehrenhaft, Theilen, and Lawrence (1958) reported a case of total anomalous venous return of the right lung with an intact atrial septum. Their case was treated with right pneumonectomy. Morrow, Awe, and Aygen (1962) reported a patient with total unilateral anomalous pulmonary venous return and intact atrial septum. This patient underwent successful total correction.

Shone and Edwards (1964) called attention to the occurrence of mitral atresia associated with anomalous pulmonary venous return and intact atrial septum. Also in 1964, Shumacker and Judd reviewed the problem of anomalous pulmonary venous drainage with the presence of an intact atrial septum and discussed the surgical correction of this lesion. These lesions have been further discussed by Snellen and co-workers (Snellen, van Ingen, and Hoefsmit, 1968) and these authors reported two patients with intact atrial septum and anomalous venous return of the right lung. Aldridge and Wigle (1965) reported a case of mitral stenosis in association with partial anomalous pulmonary venous drainage and intact atrial septum, but the patient died before operation could be undertaken. Their view of the literature revealed 10 similar cases.
However, following Gilman and his co-workers' report (1957) of anomalous drainage associated with mitral stenosis, no successful repair was reported until Adler and associates (Adler, Berger, Starkey, and Abelmann, 1964) had a patient who underwent successful repair of the anomalous drainage and correction of mitral stenosis by commissurotomy. Reid and co-workers (Reid, Barclay, Stevenson, Welsh, and McSwan, 1968) reported a case of mitral stenosis in association with partial anomalous venous return. Again, the mitral stenosis was treated with commissurotomy.

The present report concerns a patient with total anomalous pulmonary venous drainage of the right lung with intact atrial septum and calcific mitral stenosis. This unusual combination of anomalies results in each lung having a different pulmonary blood flow and vascular resistance. Following correction of the anatomical defect, changes in distribution of pulmonary blood flow were studied. This probably represents the first reported case of total anomalous pulmonary venous drainage of the right lung with intact atrial septum and associated mitral stenosis completely corrected using a prosthetic mitral valve.

\section{CASE REPORT}

M. J., a 47-year-old white man, was rejected by the Armed Forces in 1942 because of a heart murmur detected during routine induction physical examination. No symptoms were noted until three years before admission, at age 44 , when he noted exertional dyspnoea. One year before admission he had had a brief episode of pedal oedema. Three weeks before 
admission he noted a recurrence of oedema without paroxysmal nocturnal dyspnoea. orthopnoea, or chest pain. There had been no history of cyanosis or syncopal episodes. He was referred for evaluation and cardiac catheterization.

Physical examination revealed a well-developed man without cyanosis. The blood pressure was $130 / 80$ $\mathrm{mm}$. $\mathrm{Hg}$, pulse 80 , and there was normal sinus rhythm. The lungs were clear; there was an accentuated A-wave at the jugular venous pulse. Palpable maximal impulse was diffusely present in the fifth and sixth intercostal spaces at the mid-clavicular line. There were no thrills; the first heart sound was increased, the second heart sound had a wide split. There were both atrial and ventricular gallops at the apex. There was a grade II to VI pansystolic murmur at the apex. There was a diastolic apical murmur when the patient was in the left lateral decubitus position. The abdomen was soft, the liver was not enlarged, and there was no peripheral oedema. At this time the haemoglobin was 14.9 g. $/ 100 \mathrm{ml}$. An electrocardiogram revealed right axis deviation. There were tall $\mathbf{P}$ waves in VI with a duration of 0.13 second. The $R$ wave in VI was $7 \mathrm{~mm}$. with $P$-wave inversion across the entire precordium except V6.
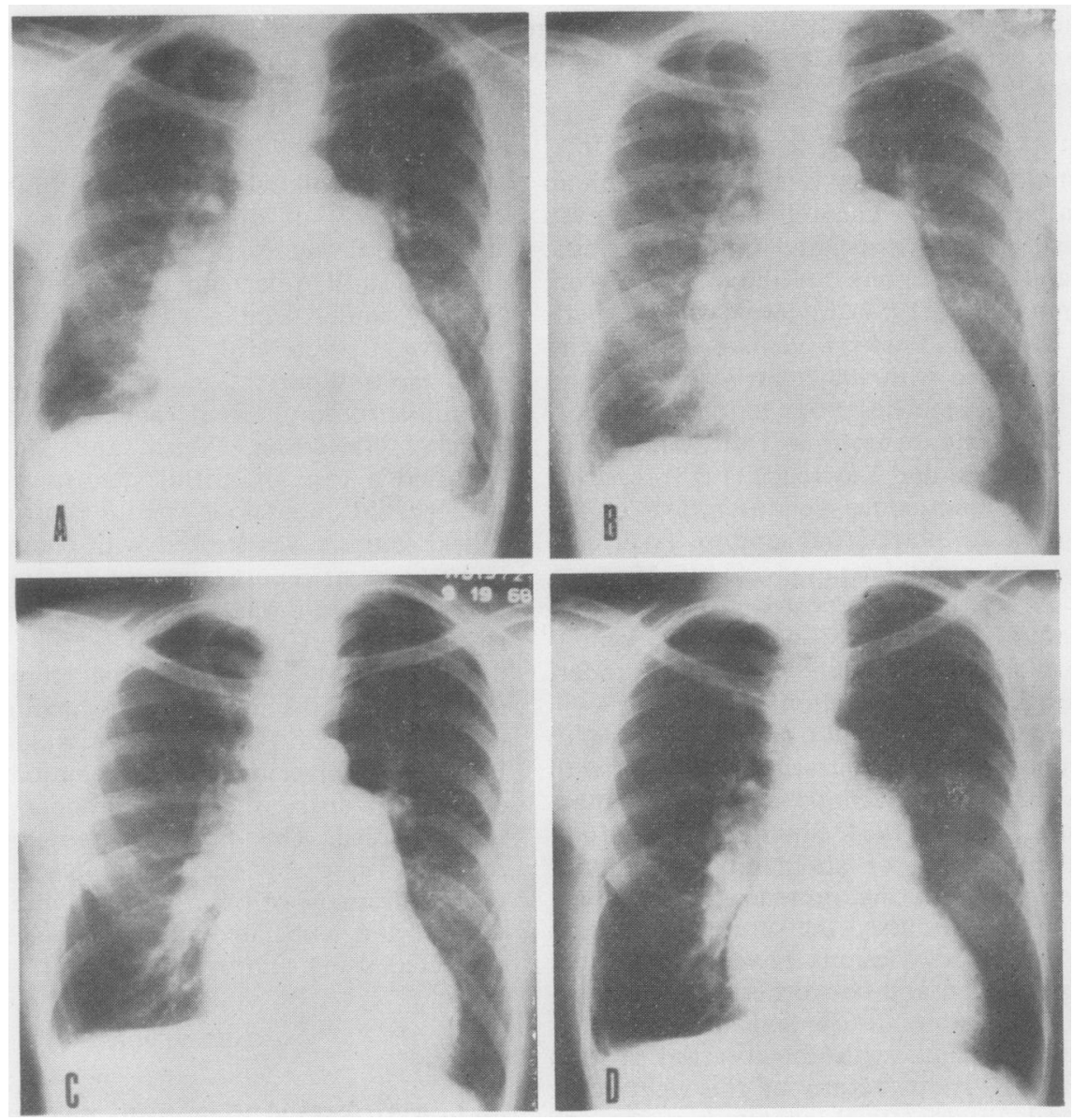

FIG. 1. Sequential series of chest radiographs $(A)$ preoperative, $(B)$ immediately after surgery, $(C)$ six months after operation, and $(D)$ one year following total correction. Note the decrease in heart size and reduction in size of the right pulmonary artery. Lung markings are reduced bilaterally. 


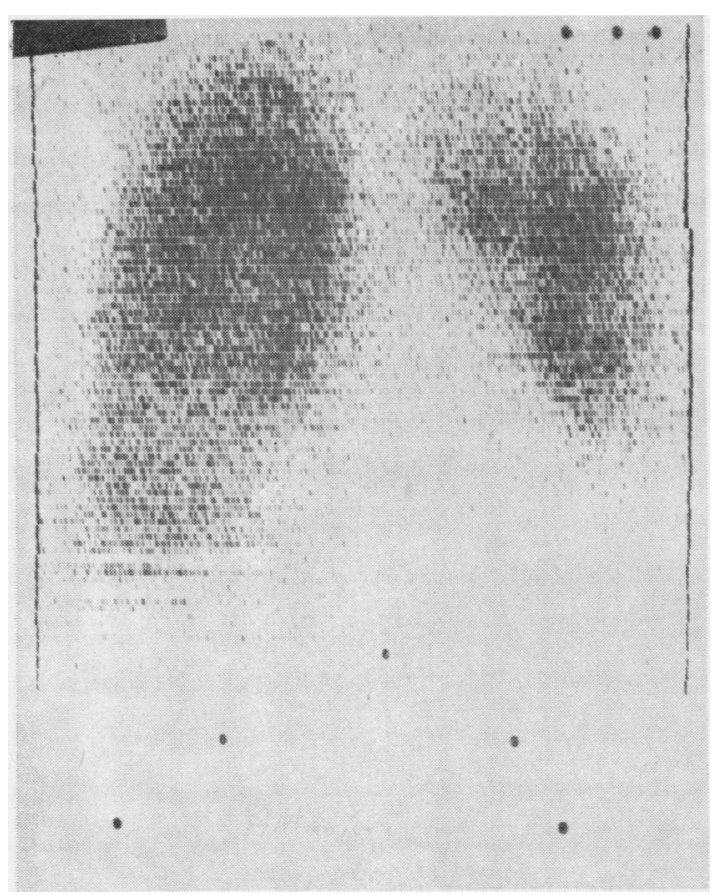

FIG. 2. Routine anterior lung scan obtained one year after operation at the time of the autofluoroscopic quantitation of distribution of blood flow. Distribution of flow was considered normal at the time of this study.

A chest radiograph showed a markedly enlarged central pulmonary artery. The peripheral pulmonary vasculature appeared to be within normal limits. There was gross cardiomegaly with right atrial and right ventricular enlargement and the left atrium was also markedly enlarged. Fluoroscopy demonstrated calcification of the mitral valve. The pu'monary artery had prominent pulsations. Cardiac catheterization showed a pulmonary artery pressure of $112 / 36$ (61) $\mathrm{mm}$. $\mathrm{Hg}$ and an aortic pressure of 142/83 (105) mm. Hg. Total pulmonary blood flow was $11.51 . /$ minute with a systemic flow of $4.61 . /$ minute. Thus left lung flow was $4.6 \mathrm{l} / \mathrm{minute}$ and right lung flow 6.9 1./minute. Resistance in the left lung was calculated as 15 Wood units and in the right lung as 5 Wood units. Cineangiography showed the pulmonary artery to be markedly enlarged and there was filling of the right atrium and inferior vena cava by this injection. The atrial septum appeared intact. It was considered that this patient had moderate mitral stenosis with a diastolic gradient of $11 \mathrm{~mm}$. $\mathrm{Hg}$ and a valve area calculated at $1.0 \mathrm{~cm}^{2}$. No mitral insufficiency was seen.

The preoperative diagnosis was mitral stenosis associated with anomalous pulmonary venous return from the right lung and intact atrial septum. At surgery a median sternotomy approach was used and total cardiopulmonary bypass was begun. The right atrium was opened and two large pulmonary veins entered above the inferior vena caval junction. The septum, confirmed as intact, was released from its posterior attachment to the atrial wall and the left atrium was entered. The heart was fibrillated electrically and the mitral valve was opened with a dilator. A $3.0 \mathrm{~cm}$. opening was accomplished but the leaflets were quite stiff and calcified. It was decided to proceed with replacement of the mitral valve, and thus a medium-size Beall valve was sutured in place. A piece of Teflon felt was sutured to the posterior rim of the atrial septum and to the lateral wall of the right atrium so that the right anomalous pulmonary veins would enter the left atrium. The patient had an uncomplicated post-operative course. The sequential series of chest radiographs are shown (Fig. 1). One year after operation the distribution of pulmonary blood flow was measured using a radioauto-fluoroscope. Flow to each lung was within normal limits and a routine perfusion scan is illustrated (Fig. 2).

\section{DISCUSSION}

In the case presented, the diagnosis of total unilateral anomalous venous drainage of the right lung into the right atrium associated with calcific mitral stenosis was established at the time of cardiac catheterization. It can be seen that each lung in this anomaly has a different pulmonary flow, is subjected to a different pulmonary venous pressure and has a separate pulmonary vascular resistance. Symptoms of right-sided cardiac failure may have resulted from an increase in shunt flow as mitral stenosis became more severe and resistance in the left lung increased. Following total anatomical correction, cardiac output should be distributed equally to both lungs. However, because of the high resistance of the left lung, it appears that blood flow would be shunted away from the left pulmonary artery because of the low resistance in the right side. By one year after correction, the distribution of blood flow to the lungs appears to be essentially normal. However, the chest film (Fig. 1) suggested an increased flow to the right lung for six months after operation. In the immediate post-operative period, there was an increase in the prominence of the pulmonary artery and vascular markings on the right. One year after correction the chest films demonstrated an increase in the prominence of the pulmonary vasculature on the left, the right pulmonary artery had become much less prominent on the chest radiograph, and the heart size was markedly reduced. 


\section{REFERENCES}

Adler, L. N., Berger, R. L., Starkey, G. W. B., and Abelmann, W. H. (1964). Anomalous venous drainage associated with mitral stenosis. New Engl. J. Med., 270, 166.

Aldridge, H. E., and Wigle, E. D. (1965). Partial anomalous pulmonary venous drainage with intact interatrial septum associated with congenital mitral stenosis. Circulation, 31, 579.

Ehrenhaft, J. L., Theilen, E. O., and Lawrence, M. S. (1958). The surgical treatment of partial and total anomalous pulmonary venous connections. Ann. Surg., 148, 249.

Gilman, R. A., Skowron, C. A. R., Musser, B. G., and Bailey, C. P. (1957). Partial anomalous venous drainage. Amer. J. Surg., 94,688 .
Morrow, A. G., Awe, W. C., and Aygen, M. M. (1962). Total unilateral anomalous pulmonary venous connection with intact atrial septum. Amer. J. Cardiol., 9, 933.

Reid, J. M., Barclay, R. S., Stevenson, J. G., Welsh, T. M. and McSwan, N. (1968). Rheumatic mitral stenosis in association with partial anomalous pulmonary venous return. Thorax, 23, 197.

Shone, J. D., and Edwards, J. E. (1964). Mitral atresia associated with pulmonary venous anomalies. Brit. Heart J., 26, 241.

Shumacker, H. B., and Judd, D. (1964). Partial anomalous pulmonary venous return with reference to drainage into the inferior vena cava and to an intact atrial septum. J. cardiovasc. Surg. (Torino), $5,271$.

Snellen, H. A., van Ingen, H. C., and Hoefsmit, E. Ch. M. (1968), Patterns of anomalous pulmonary venous drainage. Circulation 38,45 . 